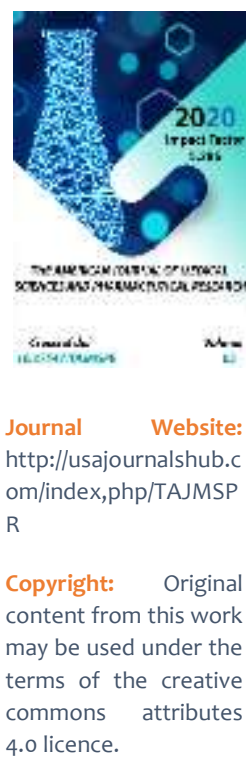

\title{
Clinical And Electro-Neuromyographic Changes In The Pathology Of The Muscular System In Patients With Hemophilia
}

\author{
Madasheva Anazhan Gazkhanovna \\ Assistants Of The Department Of Hematology, Samarkand Medical Institute, Uzbekistan \\ Mamatkulova Feruza Khaidarovna \\ Assistants Of The Department Of Hematology, Samarkand Medical Institute, Uzbekistan \\ Uralov Rustam Sherbek Ugli \\ Assistants Of The Department Of Hematology, Samarkand Medical Institute, Uzbekistan
}

\section{ABSTRACT}

Electro-neuromyographic examination allows you to accurately assess muscle activity and pathogenetic changes in nerve tissues. When compression and injuries of the musculo-nervous system application based on electro stimulation is widespread and quite reasonable and is considered a highly effective method. The use of electrical stimulation in in patients with hemophilia improves muscles and peripheral nerves microcirculation and metabolism.

\section{KEYWORDS}

Hemophilla electro-neuromyographic, clinical neurological changes, electroneuromyography.

\section{INTRODUCTION}

Blood has always been linked to life one cannot live without blood. Blood losses are not only provoked; there are those related to blood abnormalities. Hemophilia is one of them, a constitutional hemorrhagic disease, serious due to risk of fatal hemorrhage, genetically transmitted in a recessive mode linked to the $X$ chromosome. This genetic abnormality, resulting in a deficiency of a coagulation factor VIII for hemophilia A or coagulation factor IX for hemophilia B, has not spared Madagascar. It affects without exception all countries in the 
world, developing or developed countries, but the level of understanding, care and evolution vary from country to country. Its prevalence is not completely known in Africa for several reasons: rarity of the disease, high cost of its management, insufficient number of specialists in hematology and absence of adequate laboratories for the biological diagnosis of this disease. It is, however, established that hemophilia is a ubiquitous condition with an annual incidence of 1 in 5000 male births and an estimated population prevalence of 1 in 10,000 to 12,000 regardless of race or geographical area. It is the most common serious hemorrhagic disease in the world. The incidence of hemophilia $A$ is 1 per 5000 births of male children while that of hemophilia B is 1 in 30,000. Hemophilia has always been present in Madagascar if we consider children who died after bleeding circumcision, as reported in the family history of the diagnosed patients, although, the 125 currently registered patients amount to $5 \%$ of the estimated number of Malagasy patient with hemophilia (PwH).

Hemophilia, a constitutional bleeding disease, has always been present in Madagascar considering children who died after bleeding circumcision, as reported in the family history of the diagnosed patients. Hemophilia is serious because of the potentially fatal risk of hemorrhage.

Cases of de novo hemophilia were found in the absence of similar hemorrhagic situations in the family. These constitute most of Malagasy $\mathrm{PwH} \mathrm{B}$, which are curiously higher in frequency compared to the theoretical world data. The $\mathrm{PwH}$ is a person who lives first in a society. Their immediate neighborhoods, which may be their family, playmates or classmates, are primarily confronted with the hemorrhagic accidents that may occur. They may also be surrounded by members of the society of hemophilia. But they will be routed when needed directly to the nearest health center and especially to the responsible for their illness if they are already known.. Treatment was based on substitution therapy, with or without adjuvant therapy. The study considered the use of fresh frozen plasma (FFP) transfusion, the use of other hemostatic drugs, and access to the specific antihemophilic factor concentrate. The study noted the evolution of the bleeding syndrome after the treatment undertaken as well as the state of the patient during his hospitalization, the favorable course, the presence of complications or the patient's death.

\section{RESEARCH RESULTS}

Clinical and neurological studies indicate that in patients with hemarthrosis, movement disorders came to the fore with limited mobility in the pathological joint with the presence of muscle atrophy working character. Muscle strength was moderately reduced, however tendon reflexes were evoked and retained in $86.5 \%$ of patients. Surveys show that a significant decrease in the maximum amplitudes of the Motivate to $4.22 \pm 0.12 \mathrm{mV}(P$ $<0.05$ for the lunar and $4.03 \pm 0.11 \mathrm{mV}(P<0.05)$ for28tibial nerves against the background of a slight decrease in the conduction speed impulse along nerve fibers up to $57.3 \pm 0.2 \mathrm{~m} / \mathrm{s}$ along the lunar and up to $46.2 \pm 0.18$ ( $P$ $<0.05)$, along the tibia nerves, which indicated a muscle component movement disorders. In 11 patients with soft tissue hemorrhages the formation of hematomas caused compression of the peripheral nerve trunks with the development of compression neuropathy . These patients had a sharp decrease in the speed of the impulse along the lunar nerve to $24.8 \pm 0.3 \mathrm{~m} / \mathrm{s}$, and tibial nerve up to $29.5 \pm 0.4$ $\mathrm{m} / \mathrm{s},(\mathrm{P}<0.05)$ with a decrease in the 
amplitudes of the $M$-response up to $2.980 \pm$ $3 \mathrm{mV}$ and $2.90 .24 \mathrm{mV} \pm(\mathrm{P}<0.05)$, respectively. This was the result compression-ischemic processes during compression of the trunks of the studied nerve she atoms and subsequent necrotic processes in soft tissues. Data electro neuromyography mean values of SPI, m / sUlnaris D $44.7 \pm 0.24$; Ulnaris S47.75 \pm 0.16 ; Medianus D $49 \pm 0.34$; Medianus S $48.6 \pm 0.16$; Radialis D $49.05 \pm 0.25$; RadialisS $48.8 \pm 0.4$; Femoralis D $46.4 \pm 0.56$; FemoralisS $45.2 \pm 0.51$; Tibialis D $46.7 \pm 0.41$ Tibialis S46.15 \pm 0.8 ; Peroneus D 47.7 \pm 0.32 ; Peroneus S $47.14 \pm 0.26$; Lat.M. ms Ulnaris D $16 \pm 0.06$; Ulnaris S $14.06 \pm$ 0.18; Medianus D 13.16 \pm 0.16; Medianus S 14.48 \pm 0.26 ; Radialis D12.9 \pm 0.28 ; Radialis S $13.4 \pm$ 0.34 ; Femoralis D $17.22 \pm 0.41$; Femoralis S18.2 \pm 0.51 ; TibialisD $16.46 \pm 0.28$; Tibialis S16.9 \pm 0.62 ; Peroneus D14.2 \pm 0.51 ; Peroneus S $14.9 \pm 0.35$; Ampl.M, mV Ulnaris D $3.74 \pm 0.12$; Ulnaris S 3.9 \pm 0.1; Medianus D 4.06 \pm 0.16 ; MedianusS3.91 \pm 0.17; Radialis D $4.06 \pm 0.16$; RadialisS $3.35 \pm 0.12$; FemoralisD $4.6 \pm 0.23$; Femoralis S4.4 \pm 0.21 ; Tibialis D $3.35 \pm 0.19$; Tibialis S $3.2 \pm 0.14$; Peroneus D 2.5 \pm 0.35 ; Peroneus S2.4 \pm 0.26 ; 10 (46\%) patients with hemarthrosis in the extremities and 11 (54\%) patients with hemorrhages in soft tissues, a therapeutic electrical stimulation. Therapeutic electrical stimulation was performed on a two-channel the Synapses apparatus using epicutaneous plates. Current $1 \mathrm{~Hz}$ at 12-13 $\mathrm{m} \backslash$ Amp in upper limbs and 17-18 $\mathrm{m}$ । amp in the lower limbs. 8-10 sessions per course treatment. To improve their muscle strength and musculoskeletal rehabilitation systems.

Electro neuro myography data mean values after 8-10 sessions therapeutic electrical stimulation. SPI, m / s-Ulnaris D $45.7 \pm 0.24$; Ulnaris S47.85 \pm 0.16 ; Medianus D $49 \pm 1.34$; Medianus S $48.8 \pm 1.16$; Radialis D $49.45 \pm 0.25$; RadialisS $48.9 \pm 0.4$; Femoralis D $47.4 \pm 0.56$; Femoralis S $46.2 \pm 0.51$; Tibialis D $47.7 \pm 0.41$
Tibialis S47.15 \pm 0.8; Peroneus D $47.7 \pm 1.32$; Peroneus S 47.34 \pm 1.26; Lat.M. ms Ulnaris D 17 \pm 0.06; Ulnaris S $15.06 \pm 0.18$; Medianus D 14.16 \pm 0.16; Medianus S $14.48 \pm 0.26$; RadialisD13.9 \pm 0.28; RadialisS $14.4 \pm 0.34$; Femoralis D $17.42 \pm$ 0.41 ; Femoralis S18.2 \pm 0.51 ; TibialisD16.46 \pm 0.28 ; Tibialis S16.9 \pm 0.62 ; Peroneus D14.8 \pm 0.51 ; Peroneus S $14.9 \pm 0.35$; Ampl.M, mV Ulnaris D $3.94 \pm 0.12$; Ulnaris $S 3.9 \pm 1.1$; Medianus D $4.36 \pm 0.16$; Medianus S $3.98 \pm 0.17$; Radialis D $4.06 \pm 0.16$; Radialis S $3.45 \pm 0.12$; Femoralis D $4.8 \pm 0.23$; Femoralis S4.6 \pm 0.21 ; Tibialis D $3.65 \pm 0.19$; Tibialis S $3.8 \pm 0.14$; Peroneus D $2.9 \pm 0.35$; Peroneus S $2.8 \pm 0.26$; The speed of propagation of excitation in the distal regions motor fibers show slight improvement in performance.

The hemophilic arthropathy affects the function of the knee joint muscles. The aim of this study was to investigate the my electrical signal of knee joint muscles in different age stages during upright standing. Surface EMG (SEMG) amplitudes of quadriceps, ham-strings and gastrocnemii were measured in 191 patients with severe hemophilia $A(n=164)$ and $B(n=27)$ while standing on an even surface. After an age-based classification of patients into the subgroups HA: 17-29 ( $\mathrm{n}=$ 37), HB: 30-39 ( $n=50), H C: 40-49(n=61)$, HD: $50-70$ in years $(n=43)$ the clinical WFH score for the ankle and knee joint was determined. To normalize the SEMG values amplitude ratios (percentage of cumulated activity) were calculated with respect to the specific limb. With increasing age, the patient showed descriptively a deterioration of the joint situation. The extensors of the knee joint reached significantly higher absolute and percentage levels in the muscle activity with increasing age $(p<0.05)$.

The absolute amplitude values of the $\mathrm{Mm}$. gastrocnemi showed no differences in the age 
groups while the relative levels were decreased. The present study shows that patients with in-creasing age and degree of hemophilic arthropathy develop a modified control strategy during upright standing, in the formof a shift from the plantar flexors to the extensors of the knee join.

\section{CONCLUSIONS}

The knowledge of hemophilia by healthcare professionals in Madagascar has significantly improved the conditions of care of patients with hemophilia. The results observed in this study show a good evolution of this care; advances have been noted but much remains to be done. The evolution was mainly related to the availability of concentrates in coagulation factors, the time taken to treat patients and the existence of specialized healthcare professional dedicated to patients with hemophilia. The development of inhibitors, however, hampers the management of hemophilia, a topic of current research in the hemophilia scientific community

\section{REFERENCES}

1. Implementing Haemophilia Care in Senegal, West Africa. Haemophilia, 2000

2. Garib F.Yu. and other Immunedependent diseases. Tashkent. 1996.

3. Dekhkanov T.D. et al. Morphology of fluorescent structures of the duodenum intestines // European research, 2019.

4. Kasimov S. et al. Haemosorption In Complex Management Of Hepatargia // The International Journal of Artificial Organs.,2013.

5. Saydullayev Z.Y. et al. Evaluating the effectiveness of minimally invasive surgical treatment of patients with acute destructive cholecystitis // The
First European Conference on Biology and Medical Sciences, 2014. 